\title{
Star clusters with dual red clumps
}

\author{
Léo Girardi, ${ }^{1}$ Stefano Rubele ${ }^{1,2}$ and Leandro Kerber ${ }^{3}$ \\ ${ }^{1}$ Osservatorio Astronomico di Padova, Italy \\ ${ }^{2}$ Dipartimento di Astronomia, Università di Padova, Italy \\ ${ }^{3}$ Universidade Estadual de Santa Cruz, Ilhéus, Brazil
}

\begin{abstract}
A few star clusters in the Magellanic Clouds exhibit composite structures in the red-clump region of their colour-magnitude diagrams. The most striking case is NGC 419 in the Small Magellanic Cloud (SMC), where the red clump is composed of a main blob as well as a distinct secondary feature. This structure is demonstrated to be real and corresponds to the simultaneous presence of stars which passed through electron degeneracy after central-hydrogen exhaustion and those that did not. This rare occurrence in a single cluster allows us to set stringent constraints on its age and on the efficiency of convective-core overshooting during main-sequence evolution. We present a more detailed analysis of NGC 419, together with a first look at other populous Large Magellanic Cloud clusters which are apparently in the same phase: NGC 1751, NGC 1783, NGC 1806, NGC 1846, NGC 1852 and NGC 1917. We also compare these Magellanic Cloud cases with their Galactic counterparts, NGC 752 and NGC 7789. We emphasise the extraordinary potential of these clusters as absolute calibration marks on the age scale of stellar populations.
\end{abstract}

Keywords. galaxies: star clusters, Magellanic Clouds, stars: evolution, Hertzsprung-Russell diagram

\section{NGC 419 and its dual red clump}

NGC 419 is a populous star cluster located to the east of the Small Magellanic Cloud (SMC)'s bar in a region relatively devoid of dust and free from contamination by the SMC field. There are two wonderful pairs of HST images of this cluster, taken in the F555W and F815W filters, originally obtained as part of programme GO-10396 (PI J. S. Gallagher) and now retrievable from the HST archive. They are shown in Figure 1. While the ACS/WFC images reveal the overall cluster structure, the ACS/HRC observations show the details of the cluster core.

The HRC images allow us to perform photometry of an impressive quality, and reveal at least two surprises: the presence of multiple main-sequence turnoffs (MMSTOs), and a composite red clump which contains a pronounced faint extension (Figure 2). Both features were noticed by Glatt et al. (2008). They tentatively interpreted the faint redclump structure as being caused by SMC field contamination. However, this explanation does not stand up to a simple star count in the neighbouring field selected from the ACS/WFC image (see Girardi et al. 2009): the expected number of field red-clump stars in the ACS/HRC area is just 4.5, while the faint red clump contains at least 50 objects (Figure 2). Assuming a Poissonian distribution for the field stars, the probability $(P)$ that these 50 stars are drawn from the SMC field alone is virtually zero $\left(P<10^{-9}\right)$.

Detached binaries cannot give rise to this faint red clump either, since any combination of single stars will cause an extension of the red clump to brighter magnitudes.

What, then, is the dual red clump of NGC 419 made of? Girardi et al. (2009) show that it is caused by the presence of stars following two very different evolutionary paths. 
In the following, we will expand their arguments a little. There are, in fact, a few different ways of looking at dual red clumps, as detailed below.

\section{Electron degeneracy on the scene}

The basic fact behind dual red clumps is the dilemma facing low- to intermediate-mass stars after exhausting their central hydrogen and departing from the main sequence: to $R G B$ or not to $R G B$, that is the question! (RGB: red-giant branch; adapted from Shakespeare 1600). Stars slightly more massive than $2 \mathrm{M}_{\odot}$ simply contract their hydrogenexhausted cores until a temperature of about $10^{8} \mathrm{~K}$ is reached. This causes core-helium ignition and the overall expansion of the star, which quickly settles in the red clump and

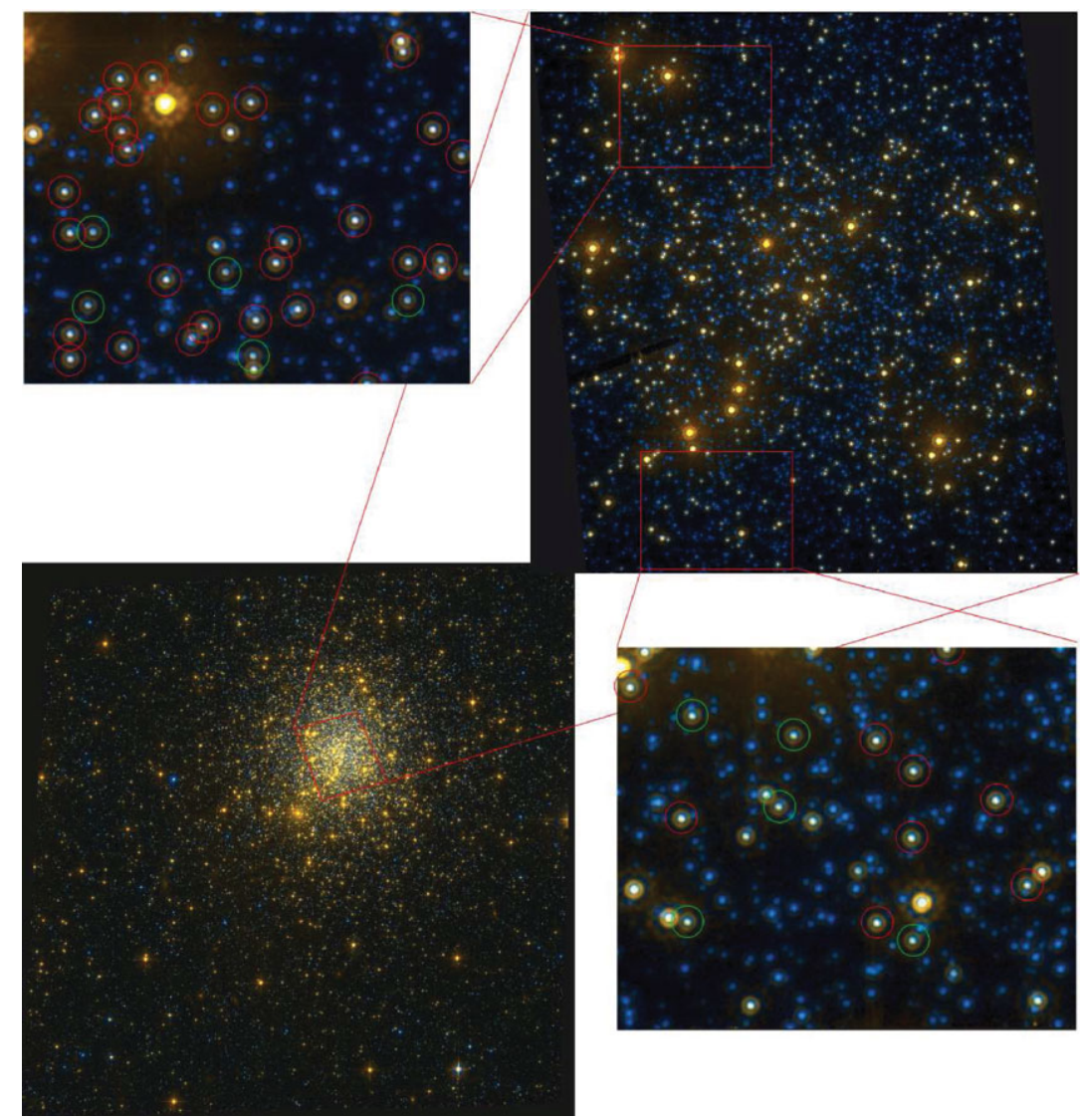

Figure 1. False-colour images (in the electronic version) of the Small Magellanic Cloud star cluster NGC 419, derived from ACS/WFC (bottom left) and HRC (top right) images in the F555W and F814W filters. The top left and bottom right panels zoom in onto the HRC image. Red-clump stars are marked with circles. At first glance, it is evident that they are quite homogeneous in their colours and luminosities, as expected for red-clump stars. However, when comparing their first Airy rings, one notices some quite subtle and systematic differences in their brightnesses. Indeed, there are two kinds of red-clump stars: the most numerous and brighter, and a subsample (about $15 \%$ of the total) of fainter 'secondary red-clump stars' (marked with red and green circles, respectively, in the electronic version). The luminosity difference between these groups is about $0.4 \mathrm{mag}$. There is also a $\sim 0.04 \mathrm{mag}$ difference in the mean colour which, however, is too small to be noticeable in the figure. The two kinds of red-clump stars are uniformly distributed across the HRC image. 
quietly burns helium thereafter. Stars slightly less massive instead suffer from electron degeneracy in the core before helium can be ignited. Electron pressure stops core contraction and the core becomes nearly isothermal. Subsequently, neutrino losses cool its very centre. The result is that He ignition is prevented for a long time. These stars have to climb all the way up to the RGB tip, where helium ignites in a flash, finally freeing their cores from electron degeneracy.

The difference between these two evolutionary paths is a quite abrupt function of initial mass (see Sweigart et al. 1990; Girardi 1999). In an interval of order $0.2 \mathrm{M}_{\odot}$, the core mass at He ignition changes from 0.46 to $0.33 \mathrm{M}_{\odot}$, depending on the presence or absence of electron degeneracy. This core-mass difference alone causes a $\sim 0.4$ mag difference in the initial luminosity of He-burning stars (with the red clump of the younger population being fainter), which is adequate to explain the two clumps of NGC 419.

The quantitative details of this explanation, based on stellar tracks and isochrones including the complete He-burning phase and computed with a fine mass resolution, can be found in Girardi et al. (2009; see also Girardi 1999).

\section{Just another manifestation of prolonged star formation}

From the discussion above, one may already obtain a hint that dual red clumps are a common feature in galaxy fields containing stars of all initial masses (ages), provided that such fine CMD features are not blurred by effects such as differential reddening, photometric errors and metallicity dispersions (Girardi 1999). Indeed, striking examples of faint secondary clumps are provided in the Hipparcos solar-neighbourhood CMD (Girardi et al. 1998) and by the CMDs of some outer Large Magellanic Cloud (LMC) fields (Bica et al. 1998; Piatti et al. 1998).

On the other hand, it is also clear that dual red clumps were not expected to be found in single star clusters, since for a single age the dispersion in turnoff masses is negligible and much smaller than the $\sim 0.2 \mathrm{M}_{\odot}$ required to explain the presence of dual red clumps.
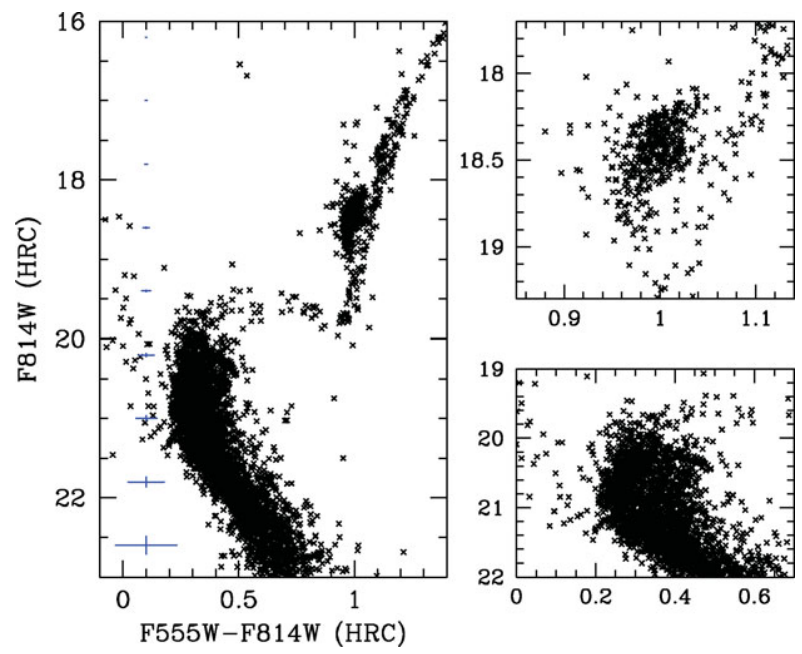

Figure 2. NGC 419 colour-magnitude diagram (CMD; left panel), derived from the ACS/HRC data (Girardi et al. 2009). The error bars in the left panel are upper limits to the photometric errors. The two right-hand panels detail the red-clump and main-sequence turnoff, clearly showing their composite structure. 
This was concluded by Girardi et al. (2000) in their study of the open clusters with dual red clumps, NGC 752 and NGC 7789.

However, we now have convincing evidence that star clusters are not single-aged stellar populations. In addition to the old globular clusters and NGC 419 itself, many intermediate-age clusters in the LMC show MMSTOs. The simplest explanation of MMSTOs is based on prolonged periods of star formation (Bertelli et al. 2003; Baume et al. 2007; Mackey et al. 2008; Milone et al. 2009).

Bastian \& de Mink (2009) recently provided an alternative explanation for the presence of MMSTOs, based on the $T_{\text {eff }} /$ colour spread of main-sequence stars with different rotation rates. The idea is, no doubt, very interesting and worth of further exploration. One crucial point to be clarified is whether rotation may cause the small spread in core mass at the end of the main sequence that is required to explain the dual red clump in NGC 419. In other words, the different rotation velocities on the main sequence have to produce a direct effect in the mass of H-exhausted cores, otherwise Bastian \& de Mink's (2009) explanation cannot be applied to NGC 419.

Rubele et al. (in prep.) go a little further into this question. They apply to NGC 419 the classical method of star-formation-history recovery through CMD reconstruction (cf. Kerber et al. 2009), deriving a star-formation rate which lasts for at least $500 \mathrm{Myr}$ (Figure 3). The spread in turnoff masses turns out to be as large as $0.18 \mathrm{M}_{\odot}$. A $\chi^{2}$-like statistic reveals that their best-fitting solutions are excellent representations of the data. It remains to be clarified whether the rotation advocated by Bastian \& de Mink (2009) will produce similarly good descriptions of the CMDs of clusters with MMSTOs, keeping at the same time the number of free parameters in the models at an acceptable level.

\section{A new way of weighing stellar cores}

The above interpretation implies that stars leaving the NGC 419 main sequence have an $\mathrm{H}$-exhausted core of almost exactly $0.33 \mathrm{M}_{\odot}$. The uncertainty in this quantity is as small as $\sim 0.01 \mathrm{M}_{\odot}$. It only depends on basic principles of stellar structure (e.g., Schönberg \&

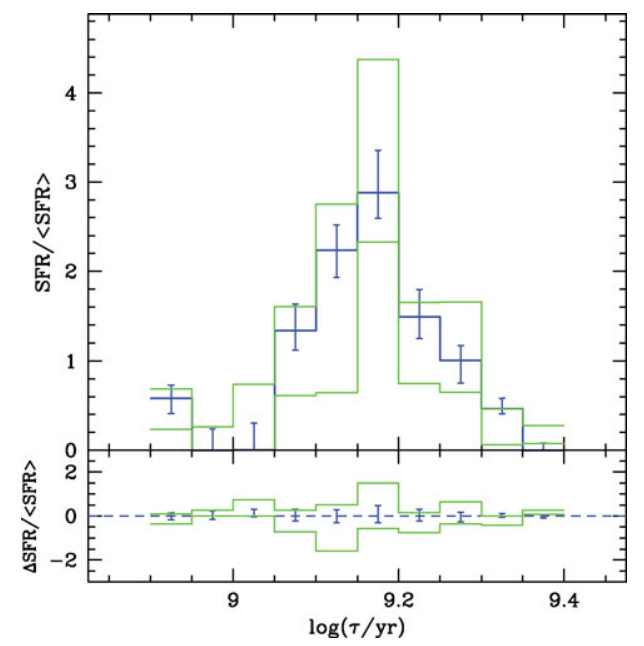

Figure 3. Star-formation history of NGC 419, as determined based on the classical method of CMD reconstruction (Rubele et al., in prep.). The heavy line with error bars is the best solution with its $68 \%$ confidence levels, reflecting random errors only. The top and bottom histograms instead indicate the total systematic errors derived from the full range of possible distances, reddenings and metallicities of the cluster. 
Chandrasekhar 1942) and on the well-known physics of partially degenerate matter. One may consider NGC 419 observations as providing the most precise (although indirect) determinations of the H-exhausted core mass of living stars.

Asteroseismology will eventually provide direct measurements of the core mass of individual red giants in the solar vicinity (see, e.g., Dupret et al. 2009), but at the cost of considerable investments in terms of dedicated telescopes and data analysis. The NGC 419 results, in contrast, come almost for free.

\section{An absolute mark on the age scale of stellar populations}

A direct consequence of knowing the core mass of a star at the end of its main-sequence evolution, is that one has a way of measuring the extent of convective-core overshooting, a phenomenum that has plagued age determinations of star clusters for decades. One just has to combine the red-clump observations (indicative of $0.33 \mathrm{M}_{\odot} \mathrm{H}$-exhausted cores) with some indicator of the main-sequence mass, which can be, very simply, the mainsequence location in the CMD. And once convective-core overshooting is constrained, age determinations become much more solid.

Girardi et al. (2009) pursued this idea by simultaneously fitting the red-clump morphology and the colour difference between the main-sequence termination and the red clump in NGC 419. The best simultaneous fit was found for a cluster mean age of $t=1.35_{-0.04}^{+0.11} \mathrm{Gyr}$ and an efficiency of convective overshooting of $\Lambda_{\mathrm{c}}=0.47_{-0.04}^{+0.14}$ pressure scale heights (see Bressan et al. 1993 for the formalism). It is worth mentioning that our results allow us to exclude the classical Schwarzschild criterion for convective boundaries with very high confidence.

It is remarkable that the above-mentioned age determination is quite independent of distance, reddening, binary fraction and overshooting (see Girardi et al. 2009). Hence, star clusters with dual red clumps have great potential of becoming absolute marks on the age scale of stellar populations.

We recall that absolute age marks are extremely rare in stellar astrophysics. Accurate absolute ages can be derived for young associations on the basis of their kinematics (for ages up to 4 Myr; e.g., Brown et al. 1997), and for old globular clusters once their upper age limit is constrained by cosmological measurements (e.g., <13.73 Gyr; Spergel et al. 2007). Inside this very wide age interval, ages of stellar aggregates depend somewhat on the extension of convective boundaries, which are notoriously uncertain from a theoretical point of view. Clusters with dual red clumps may represent the exception to this rule, providing absolute marks at ages of about 1.3 Gyr.

\section{NGC 419 is not alone}

The analysis in Girardi et al. (2009) is about to be repeated with up-to-date stellar models (Bressan et al., in prep.), and removing the assumption of a constant starformation rate during the period of formation of the MMSTOs. The error bars in the age determination will inevitably increase compared to Girardi et al. (2009). Moreover, we have recently realised that the uncertainty in the metal content represents a good fraction of the error budget for NGC 419 (Rubele et al., in prep.). More clusters with dual red clumps, with as far as possible good $[\mathrm{Fe} / \mathrm{H}]$ determinations, are necessary to obtain tighter constraints on their ages and overshooting efficiency.

Such star clusters exist and can even be numerous in the Magellanic Clouds. A quick look at the HST/ACS CMDs from Milone et al. (2009) reveals that dual red clumps are present in the LMC star clusters NGC 1751, NGC 1783, NGC 1806, NGC 1846, 
NGC 1852 and NGC 1917 (Girardi et al. 2009). All exhibit MMSTOs at a magnitude which is compatible with the lower limit for the settling of electron degeneracy. We are now examining their archival HST data, checking accurately how much of the faint clump features can be attributed to contamination from the LMC field, and then repeating the same analysis as for NGC 419.

In our Galaxy, the open clusters NGC 752 and NGC 7789 contain, among their radialvelocity members, a handful of stars which apparently belong to a faint secondary clump (Mermilliod et al. 1998; Girardi et al. 2000). Other candidates are NGC 2660, NGC 2204 (Girardi et al. 2000) and Tr 20 (Seleznev et al. 2009). In all these cases, the small numbers of red-clump stars may hamper the derivation of clear constraints to the stellar models and cluster ages. The situation with the populous Magellanic Cloud clusters is clearly different, and much more exciting.

\section{Acknowledgements}

The HST data illustrated in this talk were obtained from the Multimission Archive at the Space Telescope Science Institute (STScI). STScI is operated by the Association of Universities for Research in Astronomy, Inc., under NASA contract NAS5-26555. We thank the IAU 266 Scientific Organising Committee for the invited talk slot, I. Platais, P. Marigo, J. D. do Nascimento, G. Carraro and A. Miglio for their comments and acknowledge the support from INAF/PRIN07 CRA 1.06.10.03, contract ASI-INAF I/016/07/0, and the Brazilian agencies CNPq and FAPESP.

\section{References}

Bastian, N. \& de Mink, S. E. 2009, MNRAS (Letters), 398, L11

Baume, G., Carraro, G., Costa, E., Méndez, R. A., \& Girardi, L. 2007, MNRAS, 375, 1077

Bertelli, G., Nasi, E., Girardi, L., Chiosi, C., Zoccali, M., \& Gallart, C. 2003, AJ, 125, 770

Bica, E., Geisler, D., Dottori, H., Clariá, J. J., Piatti, A. E., \& Santos, Jr., J. F. C. 1998, AJ, 116,723

Bressan, A., Fagotto, F., Bertelli, G., \& Chiosi C. 1993, A\&AS, 100, 647

Brown, A. G. A., Dekker, G., \& de Zeeuw, P. T. 1997, MNRAS, 285, 479

Dupret M.-A., et al. 2009, A\&SA, 506, 57

Girardi, L. 1999, MNRAS, 308, 818

Girardi, L., Mermilliod, J.-C., \& Carraro, G. 2000, A\&A, 354, 892

Girardi, L., Groenewegen, M. A. T., Weiss, A., \& Salaris M. 1998, MNRAS, 301, 149

Girardi, L., Rubele, S., \& Kerber, L. 2009, MNRAS (Letters), 394, L74

Glatt, K., et al. 2008, AJ, 136, 1703

Kerber, L. O., Girardi, L., Rubele, S., \& Cioni M.-R. 2009, A\&A, 499, 697

Mackey, A. D., Broby Nielsen, P., Ferguson, A. M. N., \& Richardson J. C. 2008, ApJ (Letters), 681, L17

Mermilliod, J.-C., Mathieu, R. D., Latham, D. W., \& Mayor, M. 1998, A\&̋A, 339, 423

Milone, A. P., Bedin, L. R., Piotto, G., \& Anderson J. 2009, A\&A, 497, 755

Piatti, A. E., Geisler, D., Bica, E., Clariá, J. J., Santos Jr., J. F. C., Sarajedini, A., \& Dottori, H. $1999, A J, 118,2865$

Schönberg, M. \& Chandrasekhar, S. 1942, ApJ, 96, 161

Seleznev, A. F., Carraro, G., Costa, E., Loktin, A. V. 2010, NewA, 15, 61

Shakespeare, W., 1600, in Hamlet

Spergel D. N., et al. 2007, ApJS, 170, 377

Sweigart, A. V., Greggio, L., \& Renzini A. 1990, ApJ, 364, 527 